Revista de Matemática: Teoría y Aplicaciones 4(1): 5-19 (1997)

\title{
INTERPRETACIÓN GEOMÉTRICA DE ALGUNAS VARIEDADES DE SCHUBERT
}

\author{
Juan F. Escamilla ${ }^{1}$ - Mayra Castillo Montes $^{1}$ - Saúl Duarte Beza ${ }^{1}$
}

\begin{abstract}
Resumen
En este artículo se dan interpretaciones geométricas de las variedades de Schubert de los Grassmannianos $G(2,4), G(3,5)$ y $G(2,5)$, y algunas de sus interpretaciones en la geometría enumerativa.
\end{abstract}

\begin{abstract}
In this article we give some geometrical interpretations of the Schubert-varieties of the Grassmannians $G(2,4), G(3,5)$ and $G(2,5)$, and some of their applications to enumerative geometry.
\end{abstract}

\section{Introducción}

En este artículo se da una interpretación geométrica de las variables de Schubert de tres Grassmannianas complejas particulares: la $G(2,4)$, la $G(3,5)$ y la $G(2,5)$, las cuales juegan un papel muy importante en la geometría enumerativa, donde intervienen rectas y planos de los espacios proyectivos de dimensiones 3 y 4 respectivamente.

En la primera parte damos una reseña histórica sobre el desarrollo del álgebra y geometría que llevan al concepto de la Grassmanniana. En la segunda parte introducimos el concepto de Grassmanniana y una descripción somera de cómo resulta ésta una variedad topológica, compacta y conexa y su inmersión como subvariedad proyectiva, en un cierto espacio proyectivo, cuyas coordenadas homogéneas satisfacen las llamadas ecuaciones cuadráticas de Plücker. En la tercera parte se describen las variedades de Schubert, arriba indicadas, como las subvariedades de la Grassmanniana, que satisfacen ciertas condiciones lineales, además de las ecuaciones de Plücker correspondientes.

Finalmente se interpretan las variedades de Schubert, arriba indicadas, como ciertos conjuntos de rectas y planos proyectivos que satisfacen ciertas condiciones y se da una reseña del desarrollo del llamado cálculo de Schubert y sus aplicaciones en la resolución a problemas enumerativos.

\footnotetext{
${ }^{1}$ Licenciatura en Matemática Aplicada, Universidad de San Carlos de Guatemala, Ciudad Universitaria, zONA 12, 01012 Guatemala
} 


\section{Reseña Histórica}

En el siglo XIX se rompieron los cánones clásicos del álgebra, también a través del análisis, con criterio cada vez más abstracto de los conceptos fundamentales de la aritmética y del álgebra ordinarias, lo que dio como resultado la creación de nuevos entes que pusieron de manifiesto el carácter básico de la llamada "Ley de composición "; noción que según Bourbakí, es de las más primitivas de la matemática.

En el siglo XVIII, el auge del cálculo infinitesimal y los sucesivoos fracasos al resolver la ecuación de quinto grado por radicales, detuvieron el progreso del álgebra, pero en el siglo XIX el álgebra se dirige por distintos derroteros hacia lo que se considera hoy su problema escencial: el estudio de las estructuras algebraicas por sí mismas.

El primero de los nuevos entes es el vector, utilizado en la composición de fuerzas y de velocidades por los tratadistas de Mecánica desde fines del siglo XVII, que no tuvo repercusiones entre los matemáticos. Es posible, en cambio, que a principios del siglo XIX una especie de cálculo geométrico fuera una necesidad entre los métodos puramente sintéticos, por una parte, y los métodos analíticos vinculados a un sistema de coordenadas arbitrariamente infringido al espacio.

Gauss utiliza implícitamente la suma vectorial en su representación de los números complejos en el plano, en tanto que August Ferdinand Möbius (1790-1868) expone en 1827, un cálculo baricéntrico con importantes aplicaciones geométricas, pero en el que las coordenadas tienenn un sentido aritmético y no geométrico, y entre 1832 y 1837 Giusto Bellavitis (1803-1880) desarrolla, con su método de las equipolencias, un conjunto de operaciones con cantidades dirigidas, que equivale al cálculo vectorial de hoy.

Mientras que por un lado los vectores y sus sucesores los tensores, con el auxilio de los recursos del análisis matemático, encuentran importantes aplicaciones en diversos campos de la física, por el otro, los vectores contribuyeron a la creación de las nuevas álgebras. En ese sentido cabe señalar las obras de William Rowan Hamilton (1805-1865) y de Grassmann. Hamilton se ocupó de vectores ( el nombre es invención suya) y creó un sistema de números complejos de cuatro unidades que llamó "Quaternions" (cuaternios). Mientras la obra de Hamilton se difundió con relativa rapidez, no ocurrió lo mismo con la de Hermann Günther Grassmann (1809-1877), hombre de ciencia original, teólogo y lingüista, que a los 53 años desengañado por el escaso éxito de sus trabajos matemáticos, se dedicó al estudio del sánscrito. Su obra matemática importante es de 1844 y se conoce con el título abreviado Ausdehnungslehre (Teoría de la Extensión), aunque en su título completo se refiere a "Una nueva disciplina matemática expuesta y aclarada mediante aplicaciones". El trabajo de 1844 se refiere a la parte "lineal" de la teoría y en años posteriores publicó ampliaciones de la misma, pero la manera algo inucitada y en exceso "filosófica" para los matemáticos de la época, hizo que esta obra pasara inadvertida. Sólo más tarde, ya muerto el autor, se reconoció tanto la amplia generalidad, como la total abstracción de este cálculo algebraico-geométrico en un espacio de $n$ dimensiones, con importantes aplicaciones, y donde aparecen conceptos básicos del cálculo vectorial, como producto interno, producto externo, etc.

La obra de Grassmann fue una crítica piedra miliar en esa época de cambio en las ideas; era descrita por su autor en los siguientes términos: "Mi Ausdehnungslehre es la funda- 
mentación abstracta de la teoría del espacio, y es una disciplina puramente matemática, cuya aplicación al espacio da de sí la ciencia del espacio. Esta última ciencia, puesto que se refiere a algo dado en la naturaleza (o sea, al espacio) no es una rama de la matemática, sino una aplicación de la matemática a la naturaleza."

Como ya se dijo, la contribución de Grassmann pasó inadvertida hasta su aplicación en 1915, en la Teoría General de la Relatividad y sólo hasta fecha muy reciente, su trabajo se ha apreciado plenamente.

Es a Sylvester a quien se deben las matrices y fue Cayley, quien desarrolló, en 1858, con el cálculo de las matrices (el nombre es de él), una nueva álgebra. Con el desarrollo de la geometría, el estudio de configuraciones geométricas, como elementos de cierto espacio, conduce a identificar curvas algebraicas planas de orden $n$ con ecuaciones homogéneas de grado n; así, un primer desarrollo imporante es la geometría de rectas, debida en forma independiente a Plüker y a Cayley.

De manera que dada una recta en $\mathbb{P}^{3}$ o en forma equivalente, un plano en $\mathbb{C}^{4}$ de coordenadas homogéneas, ellos le asocian 6 determinantes de orden 2 de la matriz $A$ de la forma

$$
A=\left(\begin{array}{llll}
x_{0} & x_{1} & x_{2} & x_{3} \\
y_{0} & y_{1} & y_{2} & y_{3}
\end{array}\right)
$$

y muestran que esos seis determinantes están relacionados por medio de la ecuación

$$
P_{01} P_{23}+P_{02} P_{31}+P_{03} P_{12}=0
$$

la cual se conoce con el nombre de Ecuación de Plüker; e inversamente si 6 números, no todos cero, satisfacen dicha ecuación, esos 6 números determinan una recta en $\mathbb{P}^{3}$. Esto nos dice que el conjunto de todas las rectas puede ser visto como una subvariedad proyectiva de grado dos del espacio proyectivo $\mathbb{P}^{5}$. Luego se generalizó no solamente a rectas, sino también a subespacios $k$-dimensionales de un $n$-espacio vectorial, $k \leq n$. $\mathrm{O}$ de forma equivalente de $(k-1)$ espacios proyectivos del espacio proyectivo $\mathbb{P}^{n-1}$, dando origen a las variedades Grassmannianas.

Los $k$ menores de la matriz

$$
\left(\begin{array}{lll}
v_{11} & \cdots & v_{1 n} \\
\vdots & \ddots & \vdots \\
v_{k 1} & \cdots & v_{k n}
\end{array}\right)
$$

no son otra cosa más que las componentes del vector $\mathbf{v}_{1} \wedge \ldots \wedge \mathbf{v}_{k}$ en el espacio $\Lambda^{k} V$ introducidas por Grassmann; de ahí el nombre de Grassmanniana.

\section{$3 \quad$ La Grassmanniana $G(k, V)$}

Sea $V$ un espacio vectorial complejo de dimensión $n$. La Grassmanniana $G(k, V)$ se define como el conjunto de sub-espacios vectoriales de dimensión $k$ de $V$. Si $V=\mathbb{C}^{n}$, entonces se escribe $G(k, n)$ en lugar de $G\left(k, \mathbb{C}^{n}\right)$. Todo elemento de $G(k, n)$ se puede representar por 
medio de una matriz $A \in \mathcal{A}(k, n)$, donde $\mathcal{A}(k, n)$ es el subespacio de las matrices $k \times n$, de rango $k$, de la forma:

$$
A=\left(\begin{array}{lll}
v_{11} & \cdots & v_{1 n} \\
\vdots & \ddots & \vdots \\
v_{k 1} & \cdots & v_{k n}
\end{array}\right)
$$

Dos matrices $A$ y $A^{\prime}$ representan el mismo elemento de $G(k, n)$ si y sólo si $A=\mathbf{g} A^{\prime}$, para algún $\mathbf{g} \in G L_{k}$, donde $G L_{k}$ es el grupo de las matrices $k \times k$ invertibles.

Entonces $G(k, n)$ es el espacio de órbitas de la acción, por la izquierda, del grupo $G L_{k}$ sobre $\mathcal{A}(k, n)$.

Consideremos ahora cualquier multi-índice: $I=\left\{i_{1}, \ldots, i_{k}\right\} \subset\{1, \ldots, n\}$ de la cardinalidad $k, U_{I}:=\left\{\Lambda \in G(k, n) \mid \Lambda \cap V_{I_{0}}=\{0\}\right\}$ donde $V_{I_{0}}$ es el espacio complementario de $V_{I}=\left\langle e_{i_{1}}, \ldots, e_{i_{k}}\right\rangle$, entonces tenemos que $\Lambda \in U_{1}$ si y sólo si $\Lambda$ está representado por una sóla matriz $A, k \times n$, de rango $k$, tal que la submatriz $A^{I}$, formada por las columnas $i_{1}, \ldots, i_{k}$, es invertible, o equivalentemente, si $\Lambda$ puede ser representado de forma única por una matriz $B$ con submatriz

$$
B^{I}=\left(\begin{array}{ccc}
1 & \cdots & 0 \\
\vdots & \ddots & \vdots \\
0 & \cdots & 1
\end{array}\right)
$$

A esta representación la llamaremos la representación normalizada de $\Lambda$.

Se tiene que

$$
G(k, n)=\bigcup_{\substack{I \subseteq\{1, \ldots, n\} \\ \# I=k}} U_{I}
$$

$G(k, n)$ dotada, como espacio de órbitas, de la topología cociente respectiva, es una variedad topológica de dimensión compleja $k(n-k)$, compacta y conexa, donde $U_{I} \approx \mathbb{C}^{k(n-k)}$.

Mediante la inmersión de Plücker

$$
P: G(k, n) \longrightarrow \mathbb{P}\left(\Lambda^{k} \mathbb{C}^{n}\right)=\mathbb{P}\left(\begin{array}{c}
n \\
k
\end{array}\right)^{-1}
$$

definida por $P(\Lambda):=\mathbf{v}_{1} \wedge \ldots \wedge \mathbf{v}_{k}$, donde $\Lambda$ es el subespacio generado por los vectores linealmente independientes $\mathbf{v}_{1}, \ldots, \mathbf{v}_{k}$, la Grassmanniana $G(k, n)$ se puede ver como una subvariedad proyectiva del espacio proyectivo complejo de dimensión $N=\left(\begin{array}{c}n \\ k\end{array}\right)-1$, cuyas coordenadas homogéneas satisfacen un sistema homogéneo de ecuaciones de segundo grado con coeficientes reales, llamadas las ecuaciones de Plücker de $G(k, n)$.

Así, la $G(2,4)$ es una hipersuperficie cuadrática de $\mathbb{P}^{5}$, dada por la ecuación cuadrática:

$$
\lambda_{23} \lambda_{14}-\lambda_{13} \lambda_{24}+\lambda_{12} \lambda_{34}=0
$$


En forma similar, para el caso de la Grassmanniana $G(3,5)$, se obtienen las ecuaciones:

$$
\begin{aligned}
& \lambda_{125} \lambda_{134}-\lambda_{135} \lambda_{124}+\lambda_{123} \lambda_{145}=0 \\
& \lambda_{125} \lambda_{234}-\lambda_{235} \lambda_{124}+\lambda_{245} \lambda_{123}=0 \\
& \lambda_{135} \lambda_{234}-\lambda_{245} \lambda_{134}+\lambda_{345} \lambda_{124}=0 \\
& \lambda_{145} \lambda_{234}-\lambda_{245} \lambda_{134}-\lambda_{345} \lambda_{124}=0 \\
& \lambda_{134} \lambda_{235}-\lambda_{234} \lambda_{135}-\lambda_{345} \lambda_{123}=0 \\
& \lambda_{145} \lambda_{235}-\lambda_{245} \lambda_{135}+\lambda_{345} \lambda_{125}=0
\end{aligned}
$$

Por lo tanto, $G(3,5)$ es una variedad cuadrática de $\mathbb{P}^{9}$, de dimensión 6 .

Finalmente es de observar que cada punto $x$ de la Grassmanniana $G(k, n)$ posee una

vecindad $\mathbb{U}_{x} \stackrel{\Phi}{\approx} \mathbb{C}^{k(n-k)}$, donde cada una de las $\varphi$ es continuamente diferenciable, por consiguiente, $G(k, n)$ posee la estructura de una variedad diferenciable, incluso analítica y la inmersión de Plücker es holomorfa [6].

\section{Variedades de Schubert}

Consideremos la bandera de subespacios vectoriales:

$$
V_{1} \subseteq V_{2} \subseteq \ldots \subseteq V_{n}
$$

donde $V_{j}$ es el subespacio generado por los vectores $\mathbf{e}_{1}, \ldots, \mathbf{e}_{j}$.

Dada una sucesión de números enteros $a_{1}, \ldots, a_{k}$, se define el conjunto $W_{a_{1} \cdots a_{k}}:=\left\{\Lambda \in G(k, n) \mid \operatorname{dim}\left(\Lambda \cap V_{n-k+i-a_{i}}\right)=i \mathrm{y} \operatorname{dim}\left(\Lambda \cap V_{n-k+i-a_{i}-1}\right)=i-1\right\}$.

Si $W_{a_{1} \cdots a_{k}} \neq \emptyset$ entonces $a_{1}, \ldots, a_{k}$ debe ser una sucesión no creciente de enteros positivos, menores o iguales que $(n-k)$.

\section{Ejemplo:}

Para la Grassmanniana $G(2,4), k=2, n=4, i=1,2$ y $a_{1} \in\{0,1,2\}$, de manera que pueden definirse los siguientes conjuntos: $W_{00}, W_{10}, W_{20}, W_{21}, W_{11}, W_{22}$.

Si $\Lambda \in W_{00}$, entonces

$$
\operatorname{dim}\left(\Lambda \cap V_{3}\right)=1, \operatorname{dim}\left(\Lambda \cap V_{2}\right)=0, \operatorname{dim}\left(\Lambda \cap V_{4}\right)=2 .
$$

Si $\Lambda \in W_{10}$, entonces

$$
\operatorname{dim}\left(\Lambda \cap V_{2}\right)=1, \operatorname{dim}\left(\Lambda \cap V_{1}\right)=0, \operatorname{dim}\left(\Lambda \cap V_{4}\right)=2, \operatorname{dim}\left(\Lambda \cap V_{3}\right)=1 .
$$

Si $\Lambda \in W_{11}$, entonces

$$
\operatorname{dim}\left(\Lambda \cap V_{2}\right)=1, \operatorname{dim}\left(\Lambda \cap V_{1}\right)=0, \operatorname{dim}\left(\Lambda \cap V_{3}\right)=2 \Rightarrow \Lambda \subset V_{3}, \Lambda \neq V_{2} .
$$

Si $\Lambda \in W_{20}$, entonces

$$
\operatorname{dim}\left(\Lambda \cap V_{1}\right)=1, \operatorname{dim}\left(\Lambda \cap V_{4}\right)=2, \operatorname{dim}\left(\Lambda \cap V_{3}\right)=3 .
$$


Si $\Lambda \in W_{21}$,entonces

$$
\operatorname{dim}\left(\Lambda \cap V_{1}\right)=1, \operatorname{dim}\left(\Lambda \cap V_{2}\right)=1, \operatorname{dim}\left(\Lambda \cap V_{3}\right)=2 \Rightarrow \Lambda \subset V_{3}, \Lambda \neq V_{2} .
$$

Si $\Lambda \in W_{21}$,entonces

$$
\operatorname{dim}\left(\Lambda \cap V_{1}\right)=1, \operatorname{dim}\left(\Lambda \cap V_{2}\right)=2
$$

La clausura de la celda $W_{a_{1} \cdots a_{k}}$ viene dada por el conjunto que se define como

$$
\sigma_{a_{1} \cdots a_{k}}:=\left\{\Lambda \in G(k, n) \mid \operatorname{dim}\left(\Lambda \cap V_{n-k+i-a_{i}}\right) \geq i\right\} .
$$

El conjunto $\sigma_{a_{1} \cdots a_{k}}$ es una subvariedad cerrada de $G(k, n)$ y se llama 2 Variedad de Schubert correspondiente a $\mathbf{a}=\left(a_{1}, \ldots, a_{k}\right)$, de codimensión $\sum_{i=1}^{k} a_{i}$ en $G(k, n)$.

La condición para que un $\Lambda \in G(k, n)$ esté en uno de los subconjuntos cerrados $\sigma_{a_{1} \cdots a_{k}}$, se traduce en que sus coordenadas satisfacen, además de las ecuaciones de Grassmann, un sistema de ecuaciones lineales homogeneas: por lo tanto $\sigma_{a_{1} \cdots a_{k}}$ es una subvariedad proyectiva de $G(k, n)$.

Por otra parte, la aplicación de Plücker mapea los ciclos de Schubert de la forma

$$
\sigma_{1,0, \ldots 0}=\left\{\Lambda \in G(k, n) \mid \operatorname{dim}\left(\Lambda \cap V_{n-k}\right) \geq 1\right\}
$$

en secciones hiperplanas de $P(G(n, k)) \subset \mathbb{P}\left(\begin{array}{c}n \\ k\end{array}\right)$.

\section{Ejemplos:}

En los siguientes ejemplos, dado $\left\{i_{1}, \ldots, i_{k}\right\} \subset\{1, \ldots, n\}$, denotaremos por $\lambda_{i_{1} \cdots i_{k}}$ el determinante del menor $k \times k$ formado por las columnas $i_{1}, \ldots, i_{k}$ de la matriz que representa a $\Lambda$.

1. Para $G(2,4)$ las ecuaciones lineales homogéneas que satisface cada variedad de Schubert, además de satisfacer la ecuación de Plücker

$$
\lambda_{12} \lambda_{34}-\lambda_{13} \lambda_{24}+\lambda_{14} \lambda_{23}=0 .
$$

son las siguientes:

- Codimensión 1: $\sigma_{10}$

Por lo tanto, $\Lambda \in \sigma_{10}$ es equivalente a que $\Lambda$ satisface la ecuación 2 y además $\lambda_{34}=0$.

- Codimensión 2: $\sigma_{11}, \sigma_{20}$

a) $\sigma_{11}$

$\Lambda \in \sigma_{11}$ es equivalente a que $\Lambda$ satisface la ecuación 2 y además

$$
\lambda_{14}=0, \lambda_{24}=0, \lambda_{34}=0
$$


b) $\sigma_{20}$

$\Lambda \in \sigma_{20}$ es equivalente a que $\Lambda$ satisface la ecuación 2 y además

$$
\lambda_{23}=0, \lambda_{24}=0, \lambda_{34}=0
$$

- Codimensión 3: $\sigma_{21}$

$\Lambda \in \sigma_{21}$ es equivalente a que $\Lambda$ satisface la ecuación 2 y además

$$
\lambda_{14}=0, \lambda_{24}=0, \lambda_{34}=0, \lambda_{23}=0
$$

2. Para $G(3,5)$, las ecuaciones lineales homogéneas que satisface cada variedad de Schubert, además de satisfacer las ecuaciones cuadráticas de Plücker:

$$
\begin{aligned}
& \lambda_{125} \lambda_{134}-\lambda_{135} \lambda_{124}+\lambda_{123} \lambda_{145}=0 \\
& \lambda_{125} \lambda_{234}-\lambda_{235} \lambda_{124}+\lambda_{123} \lambda_{245}=0 \\
& \lambda_{135} \lambda_{234}-\lambda_{245} \lambda_{134}+\lambda_{124} \lambda_{345}=0 \\
& \lambda_{145} \lambda_{234}-\lambda_{245} \lambda_{134}-\lambda_{124} \lambda_{345}=0 \\
& \lambda_{235} \lambda_{134}-\lambda_{135} \lambda_{234}-\lambda_{123} \lambda_{345}=0 \\
& \lambda_{145} \lambda_{235}-\lambda_{245} \lambda_{135}+\lambda_{125} \lambda_{345}=0
\end{aligned}
$$

son las siguientes:

- Codimensión 1: $\sigma_{100} \Lambda \in \sigma_{110}$ es equivalente a que $\Lambda$ satisface las ecuaciones de Plücker 3 y además:

$$
\lambda_{345}=0
$$

- Codimensión 2: $\sigma_{110}, \sigma_{200}$

a) $\sigma_{110}$

$\Lambda \in \sigma_{110}$ es equivalente a que $\Lambda$ satisface las ecuaciones de Plücker 3 y además:

$$
\lambda_{345}=0, \lambda_{145}=0, \lambda_{245}=0
$$

b) $\sigma_{200}$

$\Lambda \in \sigma_{200}$ es equivalente a que $\Lambda$ satisface las ecuaciones de Plücker $3 \mathrm{y}$ además:

$$
\lambda_{234}=0, \lambda_{235}=0, \lambda_{245}=0 \text { y } \lambda_{345}=0 .
$$

- Codimensión 3: $\sigma_{111}, \sigma_{210}$

a) $\sigma_{111}$

$\Lambda \in \sigma_{111}$ es equivalente a que $\Lambda$ satisface las ecuaciones de Plücker 3 y además:

$$
\lambda_{345}=0, \lambda_{145}=0, \lambda_{245}=0, \lambda_{135}=0, \lambda_{125}=0, \text { y } \lambda_{235}=0
$$

b) $\sigma_{210}$

$\Lambda \in \sigma_{210}$ es equivalente a que $\Lambda$ satisface las ecuaciones de Plücker 3 y además:

$$
\lambda_{234}=0, \lambda_{235}=0, \lambda_{245}=0, \lambda_{345}=0, \lambda_{145}=0 .
$$


- Codimensión 4: $\sigma_{220}, \sigma_{211}$

a) $\sigma_{220}$

$\Lambda \in \sigma_{220}$ es equivalente a que $\Lambda$ satisface las ecuaciones de Plücker 3 y además:

$$
\lambda_{234}=0, \lambda_{235}=0, \lambda_{245}=0, \lambda_{345}=0, \lambda_{145}=0, \lambda_{135}=0, \lambda_{134}=0 .
$$

b) $\sigma_{221}$

$\Lambda \in \sigma_{221}$ es equivalente a que $\Lambda$ satisface las ecuaciones de Plücker $3 \mathrm{y}$ además:

$$
\lambda_{235}=0, \lambda_{245}=0, \lambda_{145}=0, \lambda_{345}=0, \lambda_{234}=0, \lambda_{125}=0, \lambda_{135}=0 .
$$

- Codimensión 5: $\sigma_{221}$

$\Lambda \in \sigma_{221}$ es equivalente a que $\Lambda$ satisface las ecuaciones de Plücker 3 y además:

$$
\lambda_{125}=0, \lambda_{134}=0, \lambda_{135}=0, \lambda_{145}=0, \lambda_{234}=0, \lambda_{235}=0, \lambda_{245}=0, \lambda_{345}=0 .
$$

\section{Intrepretación geométrica de algunas variedades de Schu- bert}

1. Para $G(2,4)$, se describen cuatro variedades de Schubert de codimensiones 1,2 y 3 ; además, se interpretan considerando a $G(2,4)$ como el conjunto de rectas de $\mathbb{P}^{3}$.

Las variedades de Schubert en $G(2,4)$ son: $\sigma_{10}, \sigma_{11}, \sigma_{20}$ y $\sigma_{21}$.

- Codimensión 1: $\sigma_{10}$

Si $\Lambda \in \sigma_{10}$,entonces

$$
\operatorname{dim}\left(\Lambda \cap V_{2}\right) \geq 1, \operatorname{dim}\left(\Lambda \cap V_{4}\right) \geq 2 .
$$

De manera que, considerando una recta fija $\ell_{0}$ en $\mathbb{P}^{3}, \sigma_{10}$ es el conjunto de rectas $\ell$ en $\mathbb{P}^{3}$, que intersecan $\ell_{0}$ :

$$
\sigma_{10}\left(\ell_{0}\right)=\left\{\ell \in \mathbb{P}^{3} \mid \ell \cap \ell_{0} \neq \emptyset\right\}
$$

- Codimensión 2: $\sigma_{11}$ y $\sigma_{20}$.

a) Si $\Lambda \in \sigma_{11}$, entonces

$$
\operatorname{dim}\left(\Lambda \cap V_{2}\right) \geq 1, \operatorname{dim}\left(\Lambda \cap V_{3}\right) \geq 2 \Rightarrow \Lambda \subset V_{3} .
$$

De modo que, dado el 2-plano $\mathbf{h}_{0} \subset \mathbb{P}^{3}, \sigma_{11}$ es el conjunto de las rectas $\ell$ en $\mathbb{P}^{3}$, que están contenidas en $\mathbf{h}_{0}$ :

$$
\sigma_{11}\left(\mathbf{h}_{0}\right)=\left\{\ell \in \mathbb{P}^{3} \mid \ell \subset \mathbf{h}_{0}\right\}
$$


b) Si $\Lambda \in \sigma_{20}$, entonces

$$
\operatorname{dim}\left(\Lambda \cap V_{1}\right) \geq 1 \Rightarrow V_{1} \subset \Lambda, \operatorname{dim}\left(\Lambda \cap V_{4}\right) \geq 2 .
$$

De manera que, considerando un punto fijo $p_{0}$ en $\mathbb{P}^{3}, \sigma_{20}$ es el conjunto de las rectas $\ell$ en $\mathbb{P}^{3}$, que pasan por $p_{0}$ :

$$
\sigma_{20}\left(p_{0}\right)=\left\{\ell \in \mathbb{P}^{3} \mid p_{0} \in \ell\right\} .
$$

- Codimensión 3: $\sigma_{21}$

Si $\Lambda \in \sigma_{21}$, entonces

$$
\operatorname{dim}\left(\Lambda \cap V_{1}\right) \geq 1 \Rightarrow V_{1} \subset \Lambda, \operatorname{dim}\left(\Lambda \cap V_{3}\right) \geq 2 \Rightarrow \Lambda \subset V_{3}
$$

Así que, dados un punto fijo $p_{0}$ y un 2-plano $\mathbf{h}_{0} \subset \mathbb{P}^{3}, \sigma_{21}$ es el conjunto de las rectas $\ell$ de $\mathbb{P}^{3}$, contenidas en el 2-plano $h_{0}$ que pasan por el punto $p_{0}$ :

$$
\sigma_{21}\left(p_{0}, \mathbf{h}_{0}\right)=\left\{\ell \in \mathbb{P}^{3} \mid p_{0} \in \ell \subset \mathbf{h}_{0}\right\}
$$

2. Para $G(3,5)$ se describen ocho variedades de Schubert de codimensiones $1,2,3,4$ y 5 ; además se interpretan considerando a $G(3,5)$ como el conjunto de 2-planos $\mathbb{P}^{4}$.

Las variedades de Schubert para $G(3,5)$ son: $\sigma_{100}, \sigma_{110}, \sigma_{111}, \sigma_{200}, \sigma_{210}, \sigma_{211}, \sigma_{220}, \sigma_{221}$.

- Codimensión 1: $\sigma_{100}$

Si $\Lambda \in \sigma_{100}$, entonces

$$
\operatorname{dim}\left(\Lambda \cap V_{2}\right) \geq 1, \operatorname{dim}\left(\Lambda \cap V_{4}\right) \geq 2, \operatorname{dim}\left(\Lambda \cap V_{5}\right) \geq 3 .
$$

Así que, considerando una recta fija $\ell_{0}$ contenida en el 3-plano $\mathbf{h}_{0}, \sigma_{100}$ es el conjunto de 2-planos $\mathbf{h}$ de $\mathbb{P}^{4}$, que intersecan $\ell_{0}$.

- Codimensión 2: $\sigma_{110}, \sigma_{200}$

a) $\operatorname{Si} \Lambda \in \sigma_{110}$, entonces

$$
\operatorname{dim}\left(\Lambda \cap V_{2}\right) \geq 1, \operatorname{dim}\left(\Lambda \cap V_{3}\right) \geq 2, \operatorname{dim}\left(\Lambda \cap V_{5}\right) \geq 3 .
$$

De modo que, dada una recta fija $\ell_{0}$ contenida en 2-plano $\mathbf{h}_{0}$ fijo, $\sigma_{110}$ es el conjunto de 2-planos $\mathbf{h}$ de $\mathbb{P}^{4}$, que intersecan el 2-plano $\mathbf{h}_{0}$ en una recta, la cual interseca a $\ell_{0}$ ó $\mathbf{h}=\mathbf{h}_{0}$.

b) Si $\Lambda \in \sigma_{200}$, entonces

$$
\operatorname{dim}\left(\Lambda \cap V_{1}\right) \geq 1 \Rightarrow V_{1} \subset \Lambda, \operatorname{dim}\left(\Lambda \cap V_{4}\right) \geq 2, \operatorname{dim}\left(\Lambda \cap V_{5}\right) \geq 3
$$

De manera que, considerando un punto fijo $p_{0}, \sigma_{200}$ es el conjunto de 2-planos $\mathbf{h}$ de $\mathbb{P}^{4}$, tales que $p_{0} \in \mathbf{h}$.

- Codimensión 3: $\sigma_{111}, \sigma_{210}$ 
a) Si $\Lambda \in \sigma_{111}$, entonces

$$
\operatorname{dim}\left(\Lambda \cap V_{2}\right) \geq 1, \operatorname{dim}\left(\Lambda \cap V_{3}\right) \geq 2, \operatorname{dim}\left(\Lambda \cap V_{4}\right) \geq 3 \Rightarrow \Lambda \subset V_{4} .
$$

De modo que, dado un 3-plano $\mathbf{k}_{0}$ fijo, $\sigma_{111}$ es el conjunto de 2-planos $\mathbf{h}$ de $\mathbb{P}^{4}$ contenidos en el 3-plano $\mathbf{k}_{0}$.

b) Si $\Lambda \in \sigma_{210}$, entonces

$$
\operatorname{dim}\left(\Lambda \cap V_{1}\right) \geq 1 \Rightarrow V_{1} \subset \Lambda, \operatorname{dim}\left(\Lambda \cap V_{3}\right) \geq 2, \operatorname{dim}\left(\Lambda \cap V_{5}\right) \geq 3 .
$$

Así que, considerando un punto fijo $p_{0}$ que pertenece al 2-plano $\mathbf{h}_{0}, \sigma_{210}$ es el conjunto de 2-planos $\mathbf{h}$ de $\mathbb{P}^{4}$, que intersecan el 2-plano $\mathbf{h}_{0}$ en una recta que pasa por el punto $p_{0}$ ó $\mathbf{h}=\mathbf{h}_{0}$.

- Codimensión 4: $\sigma_{220}, \sigma_{211}$

a) Si $\Lambda \in \sigma_{220}$, entonces

$$
\operatorname{dim}\left(\Lambda \cap V_{1}\right) \geq 1 \Rightarrow V_{1} \subset \Lambda, \operatorname{dim}\left(\Lambda \cap V_{2}\right) \geq 2 \Rightarrow V_{2} \subset \Lambda, \operatorname{dim}\left(\Lambda \cap V_{5}\right) \geq 3 .
$$

De manera que, dada una recta fija $\ell_{0}, \sigma_{220}$ es el conjunto de 2-planos $\mathbf{h}$ de $\mathbb{P}^{4}$ que contienen a la recta $\ell_{0}$.

b) Si $\Lambda \in \sigma_{211}$ entonces

$$
\operatorname{dim}\left(\Lambda \cap V_{1}\right) \geq 1 \Rightarrow V_{1} \subset \Lambda, \operatorname{dim}\left(\Lambda \cap V_{3}\right) \geq 2, \operatorname{dim}\left(\Lambda \cap V_{4}\right) \geq 3 \Rightarrow \Lambda \subset V_{4} .
$$

De modo que, considerando un punto fijo $p_{0}$ que pertenece a un 3-plano $\mathbf{k}_{0}$ fijo, $\sigma_{211}$ es el conjunto de 2-planos $\mathbf{h}$ de $\mathbb{P}^{4}$ que están contenidos en el 3 -plano $\mathbf{k}_{0}$ y $p_{0} \in \mathbf{h}$.

- Codimensión 5: $\sigma_{221}$

Si $\Lambda \in \sigma_{221}$ entonces

$\operatorname{dim}\left(\Lambda \cap V_{1}\right) \geq 1 \Rightarrow V_{1} \subset \Lambda, \operatorname{dim}\left(\Lambda \cap V_{2}\right) \geq 2 \Rightarrow V_{2} \subset \Lambda, \operatorname{dim}\left(\Lambda \cap V_{4}\right) \geq 3 \Rightarrow \Lambda \subset V_{4}$.

Así que, dada la recta $\ell_{0}$ contenida en un 3-plano $\mathbf{k}_{0}$ fijos, $\sigma_{221}$ es el conjunto de 2 -planos $\mathbf{h}$ de $\mathbb{P}^{4}$ contenidos en el 3 -plano $\mathbf{k}_{0}$ y que contienen a la recta $\ell_{0}$.

3. Para $G(2,5)$, se describen ocho variedades de Schubert de codimensiones 1, 2, 3, 4 y 5 ; además, se interpretan dichas variedades considerando a $G(2,5)$ como el conjunto de rectas en $\mathbb{P}^{4}$.

Las variedades de Schubert para $G(2,5)$ son: $\sigma_{10}, \sigma_{11}, \sigma_{20}, \sigma_{21}, \sigma_{22}, \sigma_{30}, \sigma_{31}, \sigma_{32}$.

- Codimensión 1: $\sigma_{10}$

Si $\Lambda \in \sigma_{10}$ entonces

$$
\operatorname{dim}\left(\Lambda \cap V_{3}\right) \geq 1, \operatorname{dim}\left(\Lambda \cap V_{5}\right) \geq 2 .
$$

Dado un 2-plano $\mathbf{h}_{0}$ de $\mathbb{P}^{4}, \sigma_{10}$ es el conjunto de rectas $\ell$ de $\mathbb{P}^{4}$ que intersecan el 2-plano $\mathbf{h}_{0}$. 
- Codimensión 2: $\sigma_{11}, \sigma_{20}$

a) Si $\Lambda \in \sigma_{11}$ entonces

$$
\operatorname{dim}\left(\Lambda \cap V_{3}\right) \geq 1, \operatorname{dim}\left(\Lambda \cap V_{4}\right) \geq 2 \Rightarrow \Lambda \subset V_{4} .
$$

Dado un 3-plano $\mathbf{k}_{0}, \sigma_{11}$ es el conjunto de rectas $\ell$ de $\mathbb{P}^{4}$ contenidas en el 3 -plano $\mathbf{k}_{0}$.

b) Si $\Lambda \in \sigma_{20}$ entonces

$$
\operatorname{dim}\left(\Lambda \cap V_{2}\right) \geq 1, \operatorname{dim}\left(\Lambda \cap V_{5}\right) \geq 2 .
$$

Dada una recta fija $\ell_{0}, \sigma_{20}$ es el conjunto de rectas $\ell$ de $\mathbb{P}^{4}$ que intersecan a la recta $\ell_{0}$.

- Codimensión 3: $\sigma_{21}, \sigma_{30}$

a) Si $\Lambda \in \sigma_{21}$ entonces

$$
\operatorname{dim}\left(\Lambda \cap V_{2}\right) \geq 1, \operatorname{dim}\left(\Lambda \cap V_{4}\right) \geq 2 .
$$

Dada una recta fija $\ell_{0}$ contenida en un 3-plano $\mathbf{k}_{0}, \sigma_{21}$ es el conjunto de rectas $\ell$ de $\mathbb{P}^{4}$ contenidas en el 3 -plano $\mathbf{k}_{0}$, que intersecan a la recta fija $\ell_{0}$.

b) Si $\Lambda \in \sigma_{30}$ entonces

$$
\operatorname{dim}\left(\Lambda \cap V_{1}\right) \geq 1 \Rightarrow V_{1} \subset \Lambda, \operatorname{dim}\left(\Lambda \cap V_{5}\right) \geq 2
$$

Dado un punto fijo $p_{0}$ en $\mathbb{P}^{4}, \sigma_{30}$ es el conjunto de rectas $\ell$ de $\mathbb{P}^{4}$ que pasan por el punto $p_{0}$.

- Codimensión 4: $\sigma_{22}, \sigma_{31}$

a) Si $\Lambda \in \sigma_{22}$ entonces

$$
\operatorname{dim}\left(\Lambda \cap V_{2}\right) \geq 1, \operatorname{dim}\left(\Lambda \cap V_{3}\right) \geq 2 \Rightarrow \Lambda \subset V_{3} .
$$

Dado un 2-plano $\mathbf{h}_{0}, \sigma_{22}$ es el conjunto de rectas $\ell$ de $\mathbb{P}^{4}$ contenidas en el 2-plano $\mathbf{h}_{0}$.

b) Si $\Lambda \in \sigma_{31}$ entonces

$$
\operatorname{dim}\left(\Lambda \cap V_{1}\right) \geq 1 \Rightarrow V_{1} \subset \Lambda, \operatorname{dim}\left(\Lambda \cap V_{4}\right) \geq 2 \Rightarrow \Lambda \subset V_{4} .
$$

Dado un punto fijo de $p_{0}$ de un 3-plano $\mathbf{k}_{0}, \sigma_{20}$ es el conjunto de rectas $\ell$ de $\mathbb{P}^{4}$ contenidas en el 3 -plano $\mathbf{k}_{0}$, que pasan por el punto $p_{0}$.

- Codimensión 5: $\sigma_{32}$

Si $\Lambda \in \sigma_{32}$ entonces

$$
\operatorname{dim}\left(\Lambda \cap V_{1}\right) \geq 1 \Rightarrow V_{1} \subset \Lambda, \operatorname{dim}\left(\Lambda \cap V_{3}\right) \geq 2 \Rightarrow \Lambda \subset V_{3} .
$$

Dado un punto fijo de $p_{0}$ de un 2-plano $\mathbf{h}_{0}, \sigma_{32}$ es el conjunto de rectas $\ell$ de $\mathbb{P}^{4}$ contenidas en el 2-plano $\mathbf{h}_{0}$, que pasan por el punto $\mathrm{p}_{0}$. 


\section{Cálculo de Schubert y geometría enumerativa}

Las variedades de Schubert constituyen la base del llamado Cálculo de Schubert.

El cálculo de Schubert fue interpretado y rigurosamente justificado por primera vez por Van der Waerden (1,929), a partir del cálculo de clases cohomológicas algebraicas desarrollado por Lefschetz.

Fue posible realizar el tratamiento algebraico de los fundamentos del cálculo de Schubert, gracias a los esfuerzos independientes de muchos matemáticos, quienes contribuyeron en la construcción de anillos de intersecciones algebraicas para reemplazar los anillos de cohomologías topológicas.

El método clásico de obtener ciertos números semejantes al número

$$
\frac{(1 ! 2 ! \cdots k ! h !)}{(n-k) ! \cdots n !}
$$

de $k$-subespacios vectoriales de un $n$ espacio vectorial, $k \leq n$, que intersecan $h=(k+1)(n-k)(n-k-1)$-subespacios generales, es debido primeramente a Schubert (1866) y fue reivindicado topológicamente por Ehresmann (1934) y algebráicamente por Hodge (1941-1942) por medio de una determinación explícita de los anillos de cohomología y, respectivamente, por la construcción de un anillo equivalente de intersecciones algebraicas sobre la Grassmanniana.

Schubert, en su libro (1879), obtiene números espectaculares de cuya validez aún no se tiene garantía.

La Geometría Enumerativa es la parte de la geometría que se interesa en saber cuántos entes geométricos, como $k$-planos, cónicas, etc., están involucrados en un cierto problema geométrico. Por ejemplo, cuántas rectas intersecan un cierto número de rectas o planos dados.

Los problemas de la geometría enumerativa reciben el nombre de Problemas Enumerativos. Teóricamente, un problema enumerativo puede resolverse siempre con ecuaciones esenciales, suponiendo que el problema puede describirse por medio de $n$ ecuaciones homogéneas en $n+1$ incógnitas homogéneas. Eliminando incógnitas, se obtiene una ecuación homogénea singular en dos incógnitas homogéneas cuyas raíces se corresponden a las raíces del sistema original. Así, el número de soluciones, contada con sus multiplicidades, si es finita, es igual al grado de la ecuación singular; y dicho grado es igual al producto de los grados de las n ecuaciones originales.

De modo que el número ponderado de soluciones se mantiene constante cuando los parámetros del problema son variables.

Hay que aclarar que la mayoría de los problemas enumerativos no pueden describirse de manera simple.

Un problema enumerativo clásico es el de encontrar el número de líneas que intersecan 4 líneas dadas en el 3-espacio proyectivo. Schubert resolvió este problema en su libro, recurriendo al Principio de Conservación del Número.

En general, los k-planos en el espacio son parametrizados por la variedad de Grass-

mann, la cual se sitúa en el $\mathrm{N}$-espacio proyectivo con $\mathrm{N}=\left(\begin{array}{c}n \\ k\end{array}\right)-1$,por medio de la llamada 
Inmersión de Plücker.

Un $k$-plano es representado por un punto, cuyas coordenadas se llaman Coordenadas de Plücker, las cuales no son arbitrarias sino que satisfacen ciertas relacciones cuadráticas descubiertas por Grassmann en 1844. Inversamente, si las relaciones cuadráticas son satisfechas por las coordenadas de un punto particular del N-espacio proyectivo, entonces el punto representa a un elemento de $G(k, n)$.

En el caso de los planos en el 4-espacio, o en forma equivalente, de rectas en el 3-espacio proyectivo, sólo una relación cuadrática es esencial. Así, $G(2,4)$ es una hipersuperficie cuadrática en el 5-espacio proyectivo.

Schubert en su libro, expresa la condición de que una línea interseca a otra línea; pero allí el se preocupaba sólo por geometría en el 3-espacio proyectivo. Posteriomente (1866), en una serie de artículos, el extendió su notación y técnica para trabajar $k$-subespacios.

Empezando con una bandera o sucesión encajada de espacios vectoriales

$$
V_{0} \subseteq V_{1} \subseteq \ldots \subseteq V_{k}
$$

él introdujo las condiciones que vimos en el capítulo anterior, conocidas como Condiciones de Schubert.

El cálculo simbólico de Schubert se desarrolló y enriqueció con muchos ejemplos, demostrando un gran potencial: en la práctica, por su aplicación en problemas enumerativos donde los métodos elementales fallaban, en la teoría, por su efecto unificador en el primer tratamiento sistemático de la geometría enumerativa.

El cálculo de Schubert se fundamenta en la idea de representar una condición geométrica sobre una figura, por un símbolo algebraico. Si los símbolos algebraicos $Y, Z$, representan condiciones independientes, entonces por definición, el producto $Y Z$ representa la condición que ambos satisfacen; y la suma $Y+Z$ representa la condición que uno u otro satisfacen. Las operaciones de adición y multipicación satisfacen las leyes de conmutatividad, asociatividad y distributividad, dotando al conjunto de dichos símbolos en una estructura de anillo. La ecuación $Y=Z$ significa que las dos condiciones son la misma para propósitos enumerativos.

La interpretación geométrica del cálculo empieza con la variedad parametrizante de las figuras de un problema enumerativo. Una condición en las figuras define un subconjunto de la variedad parametrizante, llamémoslo el subconjunto de puntos que representan las figuras que satisfacen la condición impuesta. El subconjunto es aceptable, pues condiciones aceptables son descritas por condiciones algebraicas.

Los fundamentos del cálculo de Scubert fueron trabajados primeramente por Van der Waerden, basado en la teoría de intersección topológica (simplicial) desarrollada por Lefchetz (1924), a partir de algunas ideas de Poincaré y Kronecker.

En la teoría de intersección topológica, a cada subconjunto algebraico de la variedad parametrizante se le asigna una clase cohomológica. Variando continuamente el subconjunto, se produce otro subconjunto de la misma clase cohomológica; en otras palabras, los dos subconjuntos son homológicamente equivalentes.

Si dos subconjuntos algebraicos están en posición general, entonces su intersección se asigna al producto de sus clases cohomológicas y su unión es asignada a la suma. 
Por lo tanto, si subconjuntos algebraicos distintos, en posición general, se intersecan en un número finito de puntos, éste número se conserva cuando se varían los parámetros de los subconjuntos.

Ahora bien, si los subconjuntos se definen por las condiciones de un problema enumerativo, se sigue que el número de figuras reunidas por las condiciones, se conserva cuando los parámetros del problema son variados continuamente (los números geométricos se consideran válidos sólo si son finitos).

Un tratamiento completamente algebraico de los fundamentos del cálculo, fue posible gracias al trabajo de muchos matemáticos, entre los cuales se encuentran: Severi, Van der Waerden, Chow, Chevalley, Zariski, Weil, Hodge, Gröbner, Samuel, Matsusaka, Shimura, Grothendieck, Serre, Washnitzer, Lubkin, Artin, Kleiman, Jussilla, Deligne, Berthelot, Illsie, Gersten, Quiller, Bloch, Ogus y Fulton.

Un teorema que tiene un considerable valor práctico en problemas en los que las figuras son espacios lineales, detalla la estructura aditiva y la estructura dual del anillo cohomológico de la variedad de Grassmann. Este teorema, conocido como Teorema Base, afirma que los ciclos de Schubert, esto es, las clases de cohomología de las variedades de Schubert, forman una base aditiva del anillo integral de cohomología y la base es auto-dual. Además es un anillo libre de torción [6].

El Teorema Base es esencialmente la solución de Schubert (1866) al problema de características para espacios lineales. Este teorema fue demostrado rigurosamente por primera vez por Ehresmann (1934). El observó que las variedades de Schubert proporcionan una descomposición celular de la variedad de Grassmann y derivó la estructura aditiva del anillo de cohomología.

Información acerca de la estructura multiplicativa del anillo de cohomología de la variedad de Grassmann puede ser útil para iniciar muchas investigaciones.

Dos fórmulas desarrolladas son particularmente importantes, ambas implican ciertos ciclos especiales de Schubert.

Dados dos ciclos de Schubert complementarios $\sigma_{a_{1} \cdots a_{k}}, \sigma_{b_{1} \cdots b_{k}}$, su producto de Schubert está dado por $\#\left(\sigma_{a} \cdot \sigma_{b}\right)=\sigma_{a_{1}, \ldots, a_{k}}^{n-k-b_{k}, \ldots, n-k-b_{1}}$, donde $a:=\left(a_{1}, \ldots, a_{k}\right), b:=\left(b_{1}, \ldots, b_{k}\right)$. Este producto depende sólo de la clase de cohomología de los ciclos y se suele denotar por: $\left[\sigma_{a}\right] \cdot\left[\sigma_{b}\right]$, donde los corchetes denotan las clases respectivas. Este producto es distinto de cero, si existen representantes de $\sigma_{a}$ y $\sigma_{b}$, respectivamente, que se intersecan.

La primera fórmula se conoce actualmente como fórmula de Pierri y expresa el producto de un ciclo de Schubert arbitrario con un ciclo de Schubert especial. La segunda fórmula, conocida actualmente como fórmula de Giambelli o fórmula Determinantal, expresa un ciclo de Schubert arbitrario como un deternimante de los ciclos de Schubert especiales.

La fórmula de Pierri puede usarse para encontrar el número

$$
\frac{1 ! 2 ! \cdots k ! h !}{(n-k) ! \cdots n !}
$$

de $k$ planos en el $n$-espacio que intersecan $h=(k+1)(n-k)(n-k-1)$-planos generales; dicho número es igual al grado de variedades de Schubert definida por una relación lineal en las coordenadas de Plücker, en adición a las relaciones cuadráticas definidas en la variedad 
de Grassmann $G(k, n)$. Más generalmente, la fórmula de Pierri puede usarse para encotrar el número

$$
\frac{i !}{a_{0} ! \ldots . a_{k} ! \lambda>\mu} \Pi\left(a_{\lambda}-a_{u}\right)
$$

con $i=\sum\left(a_{j}-j\right)$, de $k$-planos en el $n$-espacio que satisfacen una condición de Schubert y que tienen como dimensión los números $a_{j}$, los cuales intersecan $i(n-k-1)$-planos generales ; el número es igual al grado del producto $\left(a_{0}, \ldots, a_{k}\right) \sigma_{1}$, el cual es también el grado de la variedad de Schubert $\sigma_{a_{1} \cdots a_{k}}$.

La fórmula de Giambelli, en conjunción con el Teorema Base, implica que toda clase cohomológica es igual a una combinación lineal de productos de los ciclos de Schubert especiales; en otras palabras, los ciclos de Schubert especiales forman la base de una $\mathbb{Z}$-álgebra.

A continuación, se muestra, con algunos ejemplos, cómo pueden usarse las fórmulas de Pierri y Giambelli en la resolución de problemas enumerativos.

\section{Ejemplos:}

1. Dadas cuatro líneas de $\mathbb{P}^{3}, \ell_{1}, \ell_{2}, \ell_{3}, \ell_{4}$, en posición general, el problema consiste en determinar cuántas líneas intersecan a las cuatro líneas dadas.

Sabemos que el conjunto de líneas de $\mathbb{P}^{3}$ es la Grassmanniana de $G(2,4)$ y, en el primero de los ejemplos de la sección anterior, vimos que para una bandera proyectiva fija $p \in \ell \subset \mathbf{h}$ que consiste en de un punto, una línea y un hiperplano de $\mathbb{P}^{3}$, las variedades de Schubert son:

$$
\begin{aligned}
\sigma_{10}\left(\ell_{0}\right) & =\left\{\ell \in \mathbb{P}^{3} \mid \ell \cap \ell_{0} \neq \emptyset\right\} \\
\sigma_{20}(p) & =\left\{\ell \in \mathbb{P}^{3} \mid p \in \ell\right\} \\
\sigma_{11}(\mathbf{h}) & =\left\{\ell \in \mathbb{P}^{3} \mid \ell \subset \mathbf{h}\right\} \\
\sigma_{21}(p, h) & =\left\{\ell \in \mathbb{P}^{3} \mid p \in \ell \subset \mathbf{h}\right\}
\end{aligned}
$$

Podemos ver que el conjunto de líneas que intersecan una línea $\ell_{i}$ dada, es justamente $\sigma_{10}\left(\ell_{i}\right)$; así que la respuesta al problema viene dada por: cuatro veces el producto de $\sigma_{10}$ consigo misma, es decir $\left[\sigma_{10}\right]^{4}$. Aplicando las fórmulas de Pierri y Giambelli, se obtiene que: $\left[\sigma_{10}\right]^{4}=2$.

Por lo tanto, la respuesta al problema es: dos líneas intersecan a cuatro líneas dadas de $\mathbb{P}^{3}$ que están en posición general.

2. Dados seis 2-planos $\mathbf{h}$ de $\mathbb{P}^{4}$ en posición general, el problema consiste en determinar cuántas líneas intersecan a los seis 2-planos.

Sabemos que el conjunto de rectas de $\mathbb{P}^{4}$ es la Grassmanniana $G(2,5)$ y, en un ejemplo de la sección anterior, vimos que para una bandera proyectiva fija $p \in \ell \subset h \subset k$, que consiste de un punto, una línea, un 2-plano y un 3-plano del $\mathbb{P}^{4}$, la variedad de Schubert de codimensión uno y denotada por $\sigma_{10}$, se describió como el conjunto de rectas de $\mathbb{P}^{4}$ que intersecan a un 2-plano $\mathbf{h}$ dado. 
De manera que la solución al problema está dada por: seis veces el producto de $\sigma_{10}$ consigo misma, es decir $\left[\sigma_{10}\right]^{6}$, en $G(2,5)$.

Aplicando las fórmulas de Pierri y Giambelli, se obtiene que: $\left[\sigma_{10}\right]^{6}=5$. Por lo tanto, se obtiene como respuesta al problema que: 5 líneas intersecan seis 2-planos dados del $\mathbb{P}^{4}$ que están en posición general.

\section{Referencias}

[1] Chiavacci, R. "Qualche osservazione sui problemi totalmente reali", Pubblicazione dell'Instituto di Matematica della Università di Ferrara, Preprint 81.

[2] Chiavacci, R.; Escamilla, J. "Schubert calculus and enumerative problems", Pubblicazione dell'Instituto di Matematica della Università di Ferrara, Preprint 91.

[3] Chiavacci, R.; Escamilla, J. (1988) "Schubert calculus and enumerative problems", Bolletino U.M.I. 7 2-A: 119-126.

[4] Dieudonné, J. Abrégé D'Histoire des Mathématiques. s.l.i., s.p.i., s.f.

[5] Fulton, W. (1984) Intersection Theory. Springer-Verlag, Berlin.

[6] Griffith-Harris (1978) Introduction to Algebraic Geometry. John Wiley \& Sons, New York.

[7] Kleiman, S.L. et al.(1979) Schubert Calculus. Massachusetts Institut of Technology American Mathematical Monthly, No. 79-II.

[8] Kleiman, S.L. (1976) "Problem 15 and Rigurous Foundation of Schubert Enumerative Calculus", Proceedings of Symposia in Pure Mathematics 28.

[9] Newman, J.R. (1976) El Mundo de las Matemáticas. Vol.1. Edit. Grijalbo, México. 